Short communication

\title{
Implications of 2D Ultrasound Findings of the Scrotum in Asymptomatic Microfilaria Carriers of Wuchereria bancrofti Infection
}

\author{
Lourduraj John De Britto $^{1 *}$, Vivekanadan Ravichandran ${ }^{2}$, Lalit Kumar Das ${ }^{1}$, Subadha Prasad Pani ${ }^{1}$ \\ and Vaithialingam Govindaraj ${ }^{2}$ \\ Received 25 September, 2008 Accepted 6 February, 2009 Published online 26 March, 2009
}

\begin{abstract}
The incubation period of lymphatic filarial disease is long, and a large proportion of individuals infected with $W$. bancrofti are asymptomatic. In India, the most common presenting clinical manifestation is hydrocele of tunica vaginalis testes among males. Detection of the hydrocele in the community is considered to be a rapid assessment method for lymphatic filariasis delimitation. However, detection of early clinical manifestations is a challenge for clinicians. In the present study, the application of simple two- dimensional (2D) ultrasound procedure was explored as a way to determine the structural changes in the scrotum among male Microfilaria (Mf) carriers. Although there were no significant differences in the size of the various scrotal structures between asymptomatic Mf carriers and endemic normal subjects, sub-clinical changes in the scrotal structures and lymphatic vessel were observed in $83.3 \%$ (10/12) of asymptomatic Mf carriers. In the present communication, the therapeutic implications of the above findings are discussed.
\end{abstract}

Key words: Night blood survey, W.bancrofti, filariasis, hydrocele, microfilaria, filarial dance sign

\section{INTRODUCTION}

Despite the availability of an effective control strategy through annual mass drug administration (MDA), lymphatic filariasis continues to be a major public health problem in developing countries. Notably in India and Africa, there has been no decline in filariasis infection during the last 10 years and in many areas, there has been an increase [1]. The incubation period is long, and a large proportion of infected individuals are asymptomatic. However, the most frequent presenting clinical manifestation is hydrocele among males, and detection of hydrocele in the community is considered to be a rapid assessment method for lymphatic filariasis delimitation [2,3]. At present, case detection and individual case management appear to have secondary importance in the lymphatic filariasis elimination programme, and the detection of early clinical manifestations is a challenge for both health workers and clinicians. An alternative method is necessary, therefore, to detect the manifestations of lymphatic filariasis at a much earlier stage, to provide prompt treatment and thereby to minimize further progression of pathological changes. Earlier studies have focused on lymphatic pathology or demonstration of filarial dance sign (FDS) using pulse wave doppler [4]. With advances in ultrasound procedures, FDS could also be demonstrated using 2 D ultrasound $[5,6]$. The aim of the present study was to look comprehensively for sub-clinical manifestations of the scrotum among asymptomatic male Mf carriers using the simple 2D M-mode ultrasound procedure.

\section{PATIENTS AND METHODS}

A total of 45 W. bancrofti Mf carriers (32 males and 13 females) were detected by night blood survey (NBS) in the filarial clinic of the National Vector Borne Disease Control Programme, Pondicherry, South-India. Following the approval of the institutional human ethics committee of the Vector Control Research Centre and Government General Hospital, Pondicherry, 19 males (12 Mf carriers and 7 endemic normal subjects) in the age group of 20 to 40 years,

\footnotetext{
${ }^{1}$ Vector Control Research Centre

(Indian Council of Medical Research)

Indira Nagar, Pondicherry- 605 006, India

${ }^{2}$ Indira Gandhi Government General Hospital and Postgraduate Institute

*Corresponding author:

Tel: +91-413-2272396

E-mail: rljbritto@gmail.com
} 
who had given informed consent, were included for investigations. History of symptoms of orchitis and ADLA were recorded and clinical examination was carried out by a medical officer to detect filarial manifestations. In addition, one $\mathrm{ml}$ of venous blood was collected between 2030 and 2130 hours, the Mf count per $\mathrm{ml}$ of blood was measured by the membrane filtration technique [5], and $\mathrm{Og} 4 \mathrm{C} 3$ antigen quantification was done using a Trop-Bio commercial kit according to the manufacture's instructions. Following the guidelines of Good Clinical Diagnostics Practices, a sonologist carried out the ultrasound examination in real-time $\mathrm{M}$ mode, with a linear 7.5 $\mathrm{MHz}$ transducer using HD 11, Philips machine. Both sides of the scrotum were examined serially to identify filarial manifestations. The size of the testes and the epididymal head as well as the thickness of the tunica and scrotal skin were measured. Scrotal lymphatics were visualized for dilatation and presence of dancing adult worms. Data were entered and analyzed using SPSS 13.0, Windows application, and t-test was applied to compare the mean values of the Mf carriers and controls.

\section{RESULTS}

With the simple 2D ultrasound procedure, the mean length and width of the testes was found to be $3.94 \mathrm{~cm}( \pm$ SD 0.53) and $2.17 \mathrm{~cm}( \pm$ SD 0.48) among the Mf carriers and $4.05 \mathrm{~cm}$ ( \pm SD 0.16, $2.11 \mathrm{~cm}$ ( \pm SD 0.42) among endemic normal subjects. The mean size of the head of the epididymis among Mf carriers was $8.50 \mathrm{~mm}$ ( \pm SD 3.16) and $5.91 \mathrm{~mm}$ ( \pm SD 1.14) among endemic normal subjects. The mean thickness of the vaginal sac and the scrotal skin among Mf carriers and endemic normal subjects was 1.17 $\mathrm{mm}( \pm$ SD 0.50$)$ and $0.78 \mathrm{~mm}( \pm$ SD 0.14$)$, and $2.10 \mathrm{~mm}$ $( \pm$ SD 0.59$)$ and $2.04 \mathrm{~mm}( \pm \mathrm{SD} 0.24)$ respectively. There was no difference in length or width of the testes $(\mathrm{p}=0.602$, $p=0.806)$, thickness of the sac $(p=0.061)$ or thickness of the skin ( $p=0.796)$ between Mf carriers and endemic normals subjects. The mean size of the epididymal head was slightly larger in Mf carriers compared to endemic normal subjects, but the difference was not statistically significant $(\mathrm{p}=0.054)($ Table-1).

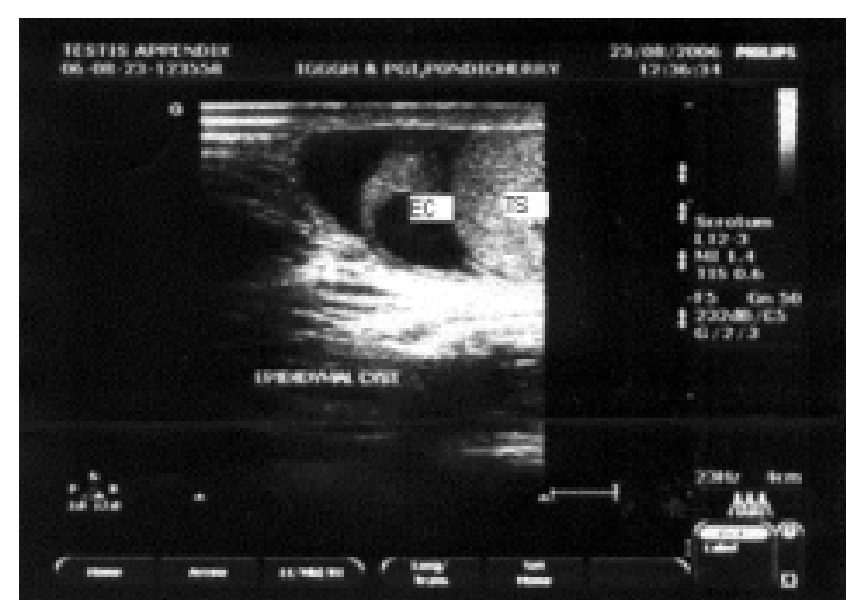

Fig: 1. Epididymal cyst (EC) seen in the head of the epididymis, the lower margin lying close to the upper border of the testis (TS)

Although there were no significant differences in the size of the different scrotal structures between asymptomatic Mf carriers and endemic normal subjects, pathological changes in the scrotal structures and lymphatic vessel were observed in $83.3 \%(10 / 12)$ of the asymptomatic Mf carriers. Sub-clinical hydrocele was observed in $66.7 \%$ (8/ 12 ) and epididymal cyst in $58.3 \%$ (7/12) of the carriers, and $41.7 \%(5 / 12)$ of the carriers had both. The other change observed was thickening of the sac of tunica in $16.7 \%(2 / 12)$ of the Mf carriers. Filarial dance sign was observed only in the high-density Mf carriers (Table-2). Presence of epididymal cyst (Figure-1) and hydrocele (Figure-2) was independent of Mf count. However, a linear 7.5 MHz transducer revealed FDS (Figure-3) only among carriers with high counts. Among the 7 endemic normal subjects, none of the above findings was noted except a solitary epididymal cyst in a 39-year-old person.

\section{DISCUSSION}

Diagnosis of scrotal manifestations of early stage $W$. bancrofti infection is a challenge considering the absence of a consensus regarding the diagnostic criteria. Cooray (1960)

Table-1: Ultrasound findings of the size of the testes, epididymal body, sac and skin thickness.

\begin{tabular}{|c|l|c|c|c|c|c|}
\hline \multirow{2}{*}{ S.no } & \multirow{2}{*}{ Parameter } & \multicolumn{2}{|c|}{$\begin{array}{c}\text { Mf carriers } \\
(\mathrm{n}=12)\end{array}$} & \multicolumn{2}{c|}{$\begin{array}{c}\text { Endemic normal } \\
(\mathrm{n}=7)\end{array}$} & \multirow{2}{*}{ p-value } \\
\cline { 3 - 6 } & & Mean & SD & Mean & SD & \\
\hline 1 & Testes (length) $(\mathrm{cms})$ & 3.94 & 0.53 & 4.05 & 0.17 & 0.602 \\
\hline 2 & Testes (width) $(\mathrm{cms})$ & 2.17 & 0.48 & 2.11 & 0.42 & 0.806 \\
\hline 3 & Epididymal body (mm) & 8.50 & 3.16 & 5.91 & 1.14 & 0.054 \\
\hline 4 & Sac thickness (mm) & 1.17 & 0.50 & 0.78 & 0.14 & 0.061 \\
\hline 5 & Skin thickness $(\mathrm{mm})$ & 2.10 & 0.59 & 2.04 & 0.24 & 0.796 \\
\hline
\end{tabular}


Table-2: Sub-clinical manifestations of the scrotum among mf carriers and endemic normal subjects by ultrasound

\begin{tabular}{|c|c|c|c|c|c|c|c|c|c|c|}
\hline \multirow[b]{2}{*}{ 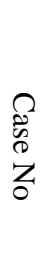 } & \multirow[b]{2}{*}{$\underset{\substack{p \\
0}}{\overrightarrow{0}}$} & \multirow[b]{2}{*}{$\stackrel{3}{\stackrel{3}{\Xi}}$} & \multirow[b]{2}{*}{ 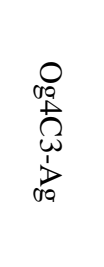 } & \multicolumn{7}{|c|}{ Parameters of scrotal manifestation } \\
\hline & & & & 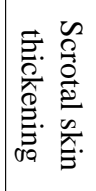 & 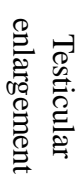 & 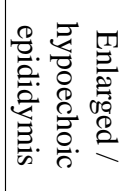 & 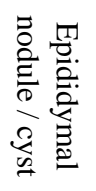 & 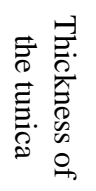 & $\begin{array}{l}\mathbb{T} \\
\stackrel{2}{0} \\
0 \\
\frac{0}{0} \\
0\end{array}$ & 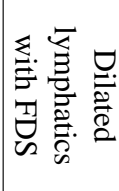 \\
\hline 1 & 21 & 22 & 39038 & - & + & + & + & - & + & - \\
\hline 2 & 21 & 496 & 23904 & - & - & - & + & - & - & + \\
\hline 3 & 22 & 3 & 36684 & + & - & - & + & + & - & - \\
\hline 4 & 24 & 0 & 386 & - & - & - & - & - & - & - \\
\hline 5 & 26 & 833 & 32296 & - & - & - & - & - & ++ & + \\
\hline 6 & 29 & 15 & 12260 & - & - & - & ++ & - & + & - \\
\hline 7 & 39 & 0 & 186 & - & - & - & + & - & - & - \\
\hline 8 & 42 & 0 & 234 & - & - & - & - & - & - & - \\
\hline 9 & 29 & 9 & 14567 & - & - & - & - & - & - & - \\
\hline 10 & 30 & 17 & 14274 & - & - & + & - & - & + & - \\
\hline 11 & 36 & 18 & 25257 & - & - & - & + & - & ++ & - \\
\hline 12 & 40 & 48 & 13902 & - & - & - & + & + & ++ & - \\
\hline 13 & 29 & 0 & 304 & - & - & - & - & - & - & - \\
\hline 14 & 38 & 406 & 15284 & - & - & - & + & - & + & + \\
\hline 15 & 37 & 151 & 18576 & - & - & - & - & - & + & - \\
\hline 16 & 31 & 0 & 282 & - & - & - & - & - & - & - \\
\hline 17 & 46 & 32 & 23962 & - & - & - & - & - & - & - \\
\hline 18 & 27 & 0 & 186 & - & - & - & - & - & - & - \\
\hline 19 & 36 & 0 & 289 & - & - & - & - & - & - & - \\
\hline
\end{tabular}

+ Unilateral, ++ Bilateral

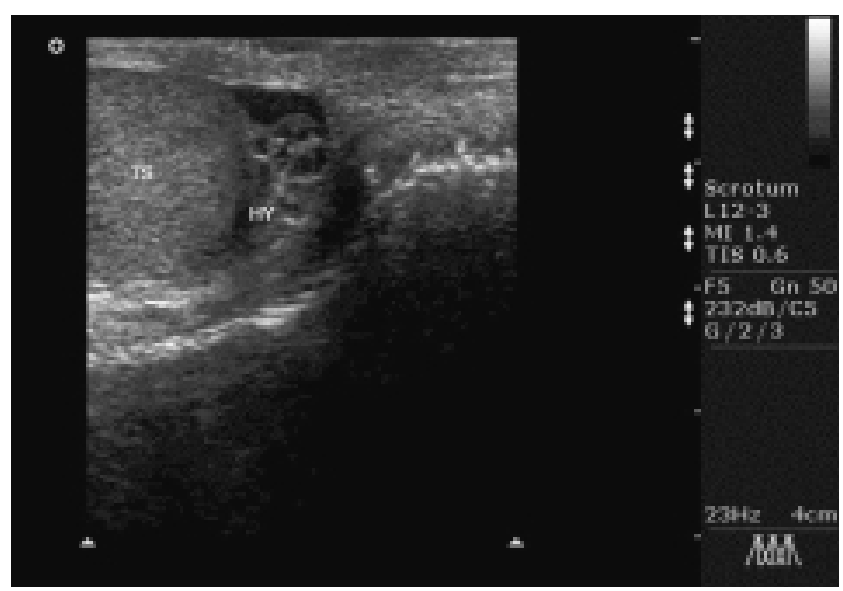

Fig: 2. Presence of minimal hydrocele (HY) in a 22-year-old Mf carrier

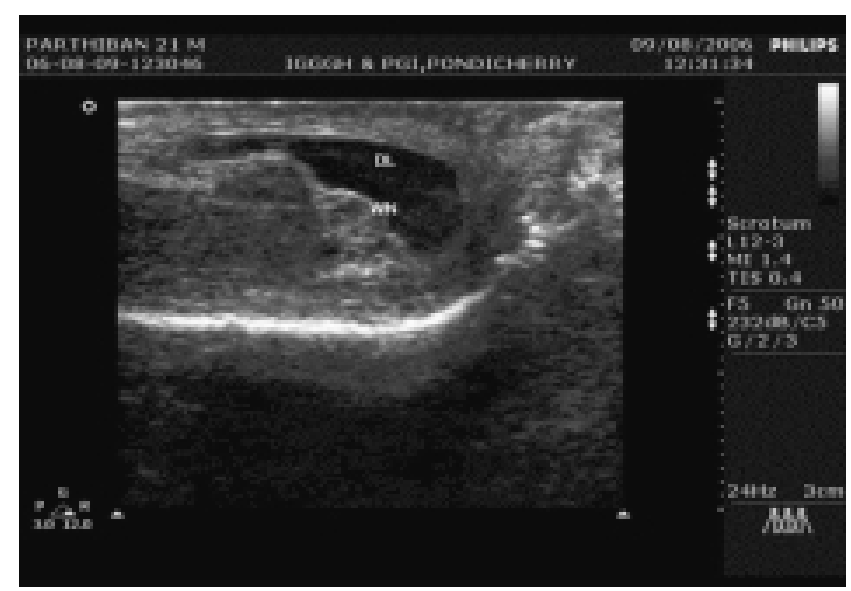

Fig: 3. 2D Ultrasound examination with a linear 7.5 MHz transducer shows the presence of the worm nests $(\mathrm{WN})$ in a dilated lymphatic vessel (DL) at the base of the scrotum. 
demonstrated that only ten out of forty eight cases of histopathologically confirmed filarial disease had been clinically suspected [7]. Pulse wave doppler disclosed small nests [8], $7.5 \mathrm{MHz}$ probes identified nests as small as $0.04 \mathrm{~cm} 2$ [9], and high-resolution ultrasound $(10 \mathrm{MHz})$ demonstrated movements of microfilaria [10]. Faris et al observed dancing worms and sub-clinical hydrocele in seven and eight of 19 clinically normal Mf carriers, respectively [4]. In community cross-sectional surveys, sub-clinical hydrocele was observed in $25.3 \%$ and $15.5 \%$ of the examined males in the high and low endemic community, respectively [11]. Apart from FDS and scrotal lymphatic pathology, sub-clinical manifestations provide an important insight into the possible clinical outcome. Examination for scrotal manifestations was considered important, because hydrocele is the most common manifestation of filarial disease and the pathology is fairly well confined. In the present study, the search for changes in various structural components of the scrotum revealed only marginal differences in the size of the epididymal head and the thickness of the vaginal sac between Mf carriers and the endemic normal subjects. However, at least two signs of scrotal manifestations were demonstrated by simple 2D ultrasound in eight out of twelve male carriers, while no sub-clinical manifestations except a solitary cyst could be seen in the endemic normal subjects. We also observed epididymal cyst in more than $50 \%$ of the Mf carriers. Although epididymal cyst is evident in other infections, no study to date has focused on the persistent cysts in different infections and the effect of treatment. In filariasis endemic areas, epididymal cyst could be an initial manifestation, reactive phenomenon or residual lesion of W.bancrofti infection. It was seen that the sub-clinical stage can be present in a wide age range and that progression of smaller to larger hydrocele occurs over the course of time [12], providing an opportunity for therapeutic intervention at an early stage. It remains unknown what proportion of the sub-clinical stage resolves on its own or precipitation of clinical disease by therapeutic intervention and the host factors responsible for these. Therapeutic intervention may prevent the development of clinical disease, its complications and costly surgical procedures. A clinical trial carried out in India showed that DEC exerts some effect on the size of the hydrocele [13]. However, the finding from Tanzania did not demonstrate any effect on the size of the hydrocele [14]. Nevertheless, these studies included large clinical forms of hydrocele, and a proportion of the cases were antigen negative. A cross - sectional population study in Africa has shown the beneficial effects on the hydrocoele prevalence and the size of the hydrocele [15]. These observations suggest that therapeutic intervention has to be initiated at the sub-clinical stage. Therefore, clinical trials are needed to determine the effi- cacy of DEC or other anti-filarial drug combinations in preventing the clinical stage and optimizing the treatment of individuals with filarial infection. The major limitations of the present observation are the small number of patients and controls and the absence of follow-up ultrasound examination. We had to restrict the number, as it was difficult to enlist endemic volunteers for ultrasound examination. Studies with larger samples and serial US examination following therapy might highlight the changes in sub-clinical manifestations over a period of time.

\section{CONCLUSION}

Sub-clinical manifestations of the scrotum in filariasis infection are common, and the current treatment schedule defined for the treatment of Mf carriers may not be sufficient in the light of ultrasound findings from this study and elsewhere. Sub-clinical scrotal manifestations provide an excellent opportunity for researchers to study the host factors and consider possible therapeutic interventions to prevent uro-genital manifestations and preclude surgery in the context of elimination of lymphatic filariasis.

\section{CONFLICT OF INTEREST: None.}

\section{ACKNOWLEDGEMENTS}

The authors thank Dr. P K Das, former Director of VCRC, Pondicherry, for providing the institutional facilities and the intramural funding from ICMR, and Dr.N.Neelamani, Assistant Director of NVBDC for providing the list of Mf carriers for the study.

\section{REFERENCES}

1 . WHO/TDR. Strategic Direction for Research: Lymphatic filariasis: February 2002; World Health Organization, Geneva; 1 -4.

2 . Gyapong JO, Webber RH, Morris J, Bennett S. Prevalence of hydrocele as a rapid diagnostic index for lymphatic filariasis. Trans R Soc Trop Med Hyg. 1998; Jan-Feb; 92(1):403.

3 . Srividya, R. Lall, K.D. Ramaiah, K.Ramu, S. L. Hoti, S. P. Pani and P. K. Das Development of rapid assessment procedures for the delimitation of lymphatic filariasis-endemic areas. Tropical Medicine and International Health volume January 2000; 5 no 1: pp 64-71.

4 . Faris R, Hussain O, El Setouhy M, Ramzy RM, Weil GJ: Bancroftian filariasis in Egypt: visualization of adult worms and subclinical lymphatic pathology by scrotal ultrasound. Am J Trop Med Hyg 1998; 59: 864-7. 
5 . Amaral F, Dreyer G, Figueredo-Silva J, Noroes J, Cavalcanti A, Samico S C, Santos A, Coutinho A: Live adult worms detected by ultrasonography in human Bancroftian filariasis. Am J Trop Med Hyg 1994; 50: 753-7.

6 . Suresh S, Kumaraswami V, Suresh I, Rajesh K, Suguna G, Vijayasekaran V, Ruckmani A, Rajamanickam MG: Ultrasonographic diagnosis of subclinical filariasis. J Ultrasound Med 1997; 16: 45-9.

7 . Cooray G H: Some observations on filarial infection in Ceylon with special reference to its histopathology. Indian Journal of Malariology 1960; 14: 617-632.

8 . Hussein O, El Setouhy M, Ahmed ES, Kandil AM, Ramzy RM, Helmy H, Weil GJ.Duplex Doppler sonographic assessment of the effects of diethylcarbamazine and albendazole therapy on adult filarial worms and adjacent host tissues in Bancroftian filariasis. Am J Trop Med Hyg. 2004; Oct; 71(4): 471-7.

9 . Reddy G S, Das L K, Pani S P, The preferential site of adult $W$ bancrofti: An ultrasound study of male asymptomatic microfilaria carriers in Pondicherry, India. The National Medical Journal of India, 2004; 17 (4): 195-196.

10 . Shyamkumar NK, Sanjeev Mehrotra, Reji Philip Athyal, Ajay Taranath, Sheila Nair, Shalini Govil, Ninan Chacko: Can We See Microfilaria On Ultrasound?: A Real-Time Ultrasound And Wet Smear Demonstration Of Dancing Microfilaria. The Internet Journal of Urology. 2004. Volume 2 Number 1.

11. Simonsen PE, Bernhard P, Jaoko WG, Meyrowitsch DW, Malecela-Lazaro MN, Magnussen P, Michael E: Filaria dance sign and subclinical hydrocoele in two east African communities with bancroftian filariasis. Trans $R$ Soc Trop Med Hyg 2002; 96: 649-53.

12 . World Health Organization: Report of an informal consultation on "Surgical approaches to the urogenital manifestations of lymphatic filariasis". WHO/CDS/CPE/CEE: 2002; 33: page 4.

13. Kar S K, Mania J: Filarial hydrocele and its treatment with DEC. Progress in Lymphology, XIV, Proceedings of the XIV International Congress of Lymphology, Washington D C.1993, 364.

14 . Bernhard P, Magnussen P, Lemnge MM: A randomized, double-blind, placebo-controlled study with diethylcarbamazine for the treatment of hydrocoele in an area of Tanzania endemic for lymphatic filariasis. Trans $R$ Soc Trop Med Hyg 2001; 95: 534-6.

15. Meyrowitsch DW, Simonsen PE, Makunde WH: Mass DEC chemotherapy for control of bancroftian filariasis: comparative efficacy of four strategies two years after start of treatment. Trans R Soc Trop Med Hyg 1996; 90: 423-8. 